\title{
CORRESPONDENCE
}

\section{Harmonizing policy on human genetic resources and benefit sharing}

\section{To the Editor:}

Failure to comprehensively define 'biological resources' in the debate regarding the international use of biologic materials is becoming an impediment to global scientific research and collaboration. The ongoing exclusion of human genetic resources (HGRs) from this debate does not reflect the realities of research in the twenty-first century, and the need for a unified regulatory policy governing the use of HGRs is becoming pressing.

The urgency of addressing international cooperation in the use of HGRs was illustrated during the recent avian influenza pandemic when Indonesia refused to release key genetic samples after Indonesian HGRs were used in vaccine development without consent, precipitating a major controversy within the World Health Organization (WHO; Geneva) and the international scientific community. The principal source of this conflict is encapsulated in the issue of access and benefit sharing (ABS). Indonesia's central concern, shared by many other developing countries, was that a product based on HGRs taken from Indonesia would not be accessible to Indonesian patients or patients in other poor countries. It is also troubling from an ethical standpoint that a product would be inaccessible to the patients donating the HGRs that made the creation of the product possible in the first place.

The key question has become how the international community defines biological resources in the context of ABS. It seems that decisions by ABS policymakers to narrow the scope of ABS provisions to plant genetic resources (PGRs) and microbial genetic resources (MGRs) were made with the understanding that HGRs would remain removed from the purview of commercial gains $^{1}$. Subsequent trends, however, unquestionably show a pattern of patenting and commercializing HGRs, highlighted by a recent ruling by the US Court of Appeals for the Federal Circuit, upholding patents on breast cancer genes held by Myriad Genetics (Salt Lake City, UT, USA) ${ }^{2}$, and this trend can be expected to grow.

Within the in vitro diagnostic industry, for example, molecular diagnostics is the fastest growing segment and is anticipated to reach a global market of $\$ 35$ billion by 2015 (ref. 3). The identification of genes that may be used in the development of in vitro diagnostics often involves a strategy of identifying genetic polymorphisms, and the comparison of different ethnic populations is an important approach to polymorphism identification often involving international collaboration in the use of HGRs. For example, a study by a French group ${ }^{4}$ compared alleles that predispose carriers to rheumatoid arthritis across ten ethnic populations from 17 different countries. It is not difficult to envision the complications that would arise if each of the 17 countries that contributed samples were to have a distinct national ABS legislative framework.

An additional factor complicating the exclusion of HGRs from ABS policies covering PGRs and MGRs is that advances in biotech are leading to increasing integration of PGRs and MGRs into the same drug discovery programs as HGRs, and even into the same molecules. It is unclear how a policy that solely governs PGRs would apply in cases where HGRs are used to identify drug targets that form the basis for drug discovery programs using molecules isolated from plant resources. A more complex example is the creation of chimeric proteins, a strategy whereby human genetic material may be directly linked to plant or microbe genetic material to generate a recombinant protein. This is a strategy that is currently being employed in antineoplastic drug development, where a human protein is combined with a plant toxin into a single molecule that selectively targets cancer cells ${ }^{5}$. In such cases, the logistics of a policy that selectively covers PGRs and MGRs become impractical.

As national governments have begun to realize the value of HGRs for technology development, their responses have varied, and the continued absence of a unified regulatory mechanism has left international scientific cooperation involving HGRs vulnerable to the vagaries of national ethical approaches and interpretations. The Indian Ministry of Health, for example, issued guidelines in 2009 regarding the restricted transfer of biological material for collaborative research ${ }^{6}$, and the Chinese Human Genetic Resources Management Office has recently revised China's policy regulating the export and import of HGRs across Chinese borders ${ }^{7}$. As more countries individually establish national regulations, the difficulties facing global $\mathrm{R} \& \mathrm{D}$ collaboration involving HGRs will expand exponentially.

The need for a unified ABS policy based on a comprehensive definition of biological resources is thus becoming urgent. Such a policy would need to provide a satisfactory balance of intellectual property rights with the establishment of viable ABS arrangements.

The most promising existing venue for the development of such a policy is the Convention on Biological Diversity (CBD). The CBD has recently adopted their most recent iteration of an international $A B S$ policy, the Nagoya Protocol. This document stipulates that any commercial benefit derived from national biological resources should recognize the supplier country and/or indigenous communities for their contributions, as well as ensuring fair and equitable sharing of benefits arising from biologic resources. Although this is obviously a major step forward in establishing an international ABS regime, it continues to exclude HGRs.

Impending legislation from around the world, coupled with the varied bilateral agreements that will inevitably form, is a recipe for confusion, acrimonious international disputes and, more importantly, a hindrance to the development of a muchneeded new generation of HGR-based pharmaceuticals. The fact that HGRs used 
in research and technology development are often obtained from indigenous communities makes the lack of an ABS regime for HGRs a matter of even greater concern, given the increased vulnerability of these populations ${ }^{8}$. The CBD currently represents the best avenue for rapidly establishing a unified international ABS policy. The proposed meeting of CBD members in Thailand this March provides an opportunity for course correction. With this next meeting of the CBD rapidly approaching, broad awareness of the importance of these issues and support from the global scientific community for the timely creation of a comprehensive policy addressing ABS in HGRs as well as in PGRs and MGRs will be critical to CBD's success.

\section{COMPETING FINANCIAL INTERESTS}

The authors declare no competing financial interests.

Sachin Chaturvedi ${ }^{1}$, Sara Crager $^{2}$, Miltos Ladikas ${ }^{3}$, Vasantha Muthuswamy ${ }^{4}$, Yeyang $\mathrm{Su}^{5}$ \& Huanming Yang ${ }^{5}$

${ }^{1}$ Research and Information System for Developing Countries (RIS), New Delhi, India.

${ }^{2}$ UCLA Medical Center, Los Angeles, California,
USA. ${ }^{3}$ University of Central Lancashire, Preston, UK. ${ }^{4}$ Formerly at Indian Council of Medical Research, New Delhi, India. ${ }^{5}$ Beijing Genomics Institute at Shenzhen, Shenzhen, GuangDong,

China.e-mail:Sachin@ris.org.in

1. Nijar, G. Statement made at the at the Seventh meeting of the Ad Hoc Open-ended Working Group on Access and Benefit-sharing: Informal Consultation with the Co-chairs and regional consultations, organized by the Secretariat of the Convention on Biological Diversity (CBD), UNESCO, Paris, 31 March-1 April, (2009).

2. Hopkins, M.M. Nat. Biotechnol. 25, 185-187 (2007).

3. Visiongain. In Vitro Diagnostics: Market Analysis 2009 -2024 (Visiongain, London, 2009). <https:// www.visiongain.com/Report/392/In-Vitro-DiagnosticsMarket-Analysis-2009-2024>

4. Barnetche, T., Constantin, A., Cantagrel, A., CambonThomsen, A. \& Gourraud, P.A. Arthritis Res. Ther. 10 R26 (2008).

5. Ben-Yehudah, A. \& Lorberboum-Galski, H. Expert Rev. Anticancer Ther. 4, 151-161 (2004).

6. ICMR. (2009) Guidelines on restricted transfer of biological material for collaborative research (Institutional Review and Ethics Committee, Indian Council of Medical Research, Indian Ministry of Health and Family Welfare, 2009)

7. Anonymous. China strengthening controls over human genetic resources (Sidley Austin, Global Life Sciences: China Update, 21 June 2011). <http://www. sidley.com/ China-Strengthening-Controls-over-Human-GeneticResources-06-21-2011/>

8. Hammond, E. \& Mayet, M. Genes from Africa: The Colonisation of Human DNA. ACB Briefing Paper, No. 5 (African Centre for Biosafety, Melville, South Africa, 2009).

\section{Strengthening African R\&D through effective transfer of tropical crop biotech to African institutions}

To the Editor:

According to a study published in your January issue $^{1}$ on genetically modified crop development and adoption in subSaharan Africa, the negative perception and limited adoption of biotech in Africa can be partly attributed to a lack of local expertise and capacity for regional development of agbiotech products. The need for selfdetermination in science is clearly emphasized in this survey. Over the past decade, training opportunities in Western laboratories have boomed for
African scholars. Supported by master's and $\mathrm{PhD}$ fellowships, African scientists now have increasing access to education in renowned Western institutions, as well as to training in cuttingedge technologies. It is widely accepted that training in the best institutions forges the scientific leaders of tomorrow who will contribute to building and strengthening of African science. Although training of African scientists abroad is essential, we argue here that building a self-sustainable African scientific workforce also requires the development of top African education and research institutions with full access to technology.

Projects such as the Next Einstein Initiative of the African Institute for Mathematical Sciences (AIMS) ${ }^{2}$ typically rely on building competence centers throughout the African continent to unlock its science potential. However, convincing the brightest and most promising African students to study in African institutions and, more ambitiously, reverse the brain drain affecting the African continent will only be possible if those institutions can offer an environment equivalent to research quality and opportunities available in Western laboratories. Access of African institutions to technology remains a prerequisite for high-quality research and for persuading the best African scientists to advance their career and research activities in Africa. In particular, technologies enabling genetic transformation and improvement of local crop production should be prioritized. These technologies are of particular benefit to the local scientific community because they can tackle problems specific to the African regional agriculture. Crop biotech resources and expertise have been listed amongst the most needed and beneficial technologies that should be rapidly transferred to African laboratories ${ }^{3}$, given their contribution to agriculture and the necessity for development of transgenic farmer-preferred crops in African laboratories, as was highlighted by Singer and colleagues ${ }^{1}$.

When the first Agrobacterium-mediated genetic transformation protocols were established for maize and cassava in 1996, the following comment was made in an editorial published in Nature Biotechnology: "It remains to be seen if, how, when and at what cost the technology will be transferred to countries where it will be of practical interest" ${ }^{\text {. }}$. Fifteen years later, efforts to establish crop transformation and biotechnology in African laboratories have started to produce tangible successes. Teams in South Africa and Uganda have recently reported the production of the first transgenic maize and banana plants entirely developed in Africa ${ }^{5,6}$. Centers of excellence, such as the Biosciences eastern and central Africa (BecA) initiative in Nairobi, have the ambition to serve as crop biotech hubs to produce transgenic African crops, such as banana, cassava and sweet potato, as well as selected African orphan crops. Even so, dissemination of crop biotech across the African continent remains a challenge. Most transformation procedures for tropical crop 